\title{
Cholesterol Modulates Amiodarone-Membrane Interactions in Model and Native Membranes
}

\author{
Maria C. Antunes-Madeira, ${ }^{1}$ Romeu A. Videira, ${ }^{1,2}$ \\ and Vítor M. C. Madeira ${ }^{*, 1}$ \\ ${ }^{1}$ Centro de Neurociências e Biologia Celular, Departamento de Zoologia, \\ Universidade de Coimbra, 3004-517 Coimbra, Portugal, \\ E-mail:vmcm@ci.uc.pt; and ${ }^{2}$ Departamento de Ambiente, \\ Escola Superior de Tecnologia, Instituto Superior Politécnico de Viseu, \\ 3500 Viseu, Portugal
}

Received June 1, 2001; Revised August 1, 2001; Accepted August 16, 2001

\begin{abstract}
The effects of cholesterol, a lipid mostly found in the sarcolemmal membranes, on the interaction of amiodarone with synthetic models of dimyristoylphosphatidylcholine (DMPC) and with native models of mitochondria and brain microsomes was studied. Alterations on the structural order of lipids were assessed by fluorescence polarization of 1,6-diphenyl1,3,5-hexatriene (DPH) probing the bilayer core, and of the propionic acid derivative 3-( $p$-(6-phenyl)-1,3,5-hexatrienyl)phenylpropionic acid (DPHPA) probing the outer regions of the bilayer. As detected by the probes and according to classic observations, cholesterol progressively increased the molecular order in the fluid phase of DMPC. Additionally, it modulated the type and extension of amiodarone effects. For low cholesterol concentrations ( $\leq 10-15 \mathrm{~mol} \%)$, amiodarone $(50 \mu \mathrm{M})$ ordered DMPC bilayers and the effects were almost identical to those observed in pure DMPC. For higher cholesterol concentrations, amiodarone ordering effects decreased slightly and faded for cholesterol concentrations as high as 25 and $30 \mathrm{~mol} \%$, when detected by DPH-PA and DPH, respectively. Above these high cholesterol concentrations, a crossover from ordering to disordering effects of amiodarone was apparent, either in the upper region of the bilayer or the hydrophobic core. The effects of amiodarone in native membranes of mitochondria and brain microsomes, in which "native" cholesterol accounts for about 0 and $25 \mathrm{~mol} \%$, respectively, correlated reasonably with the results in models of synthetic lipids. There is a close relationship between cholesterol concentration and

*Author to whom all correspondence and reprint requests should be addressed.
\end{abstract}

